\title{
Treatment of trochanteric fractures using the Gamma nail
}

Clinic of Orthopedics and Traumatology, Emergency County Hospital Constanța, Romania

\begin{abstract}
A retrospective study on 2 years and 4 months (April 2012 - August 2014) on 430 patients admitted with trochanteric fractures in our Clinic. The surgical indications depended on the patients age, degree of osteoporosis, level activity, associated pathologies and the anatomo-clinical particularity of the fractures. The fractures were operated with gamma nail. The surgery was done in the first 24-72 hours, patients were followed clinically and radiographically at 2,4 , 6 months postoperative. The age of the patients were between 28-95 years, female sex was predominant in $65 \%$ of case. It was reported a case of a fracture produced intraoperatory during progression of the nail, 11 bronhopulmonary complications, 5 phlebitis, 7 cases of hematoma after surgery, a case of pseudarthrosis with loosening of implant and 4 dead. There were no septic complications. Fractures consolidate within 1214 weeks. The results were appreciated according to fracture reduction, stability of implant, consolidation time and possibility of walking and weight bearing on operated limb.
\end{abstract}

Keywords: pertrochanteric fractures, gamma nail, surgical treatment, results

Obada Bogdan

Clinic of Orthopedics and Traumatology

Emergency County Hospital Constanța, Romania

bogdanobada@yahoo.com

\section{Introduction}

Hip fractures in the elderly are frequent, and their number is increasing fast. The trochanteric fractures pose a number of management dilemmas depending on the age, type of fractures, configuration and status of the bones. The trochanteric fractures in younger individuals are usually the result of a high-energy injury, such as a motor vehicle accident or fall from a height. Ninety percent of these fractures in the elderly result from a simple fall. The tendency to fall increases with patient age and is exacerbated by several factors, including poor vision, decreased muscle power, labile blood pressure, decreased reflexes, vascular diseases and coexisting musculoskeletal pathology $[1,2,3]$.

The paper wants to evaluate the late results of the surgical treatment of pertrochanteric fractures, using osteosynthesis with gamma nail.

\section{Material and Methods}

A retrospective study on 2 years and 4 month between April 2012 and August 2014 on 430 patients admitted with trochanteric fractures in the Clinic of 
Orthopedics and Traumatology of Emergency County Hospital Constanta Romania. The surgical indications depended on the patients age, degree of osteoporosis, level activity, associated pathologies and the anatomoclinical particularity of the fractures. We used AO classification as a criterion for the type of the fracture and it was performed with Gamma nail fixation. The Gamma nail, a combination of advantages of the sliding screw with the intramedullary nail, represents an efficient technique in the management of these fractures.

In each patient, we determined the mechanism of fracture, age, sex, surgical and inpatient time, surgical technique and complications.

\section{Results}

The age of the patients were between 28-95 years, female sex was predominant in $65 \%$ of case (Figure 1).

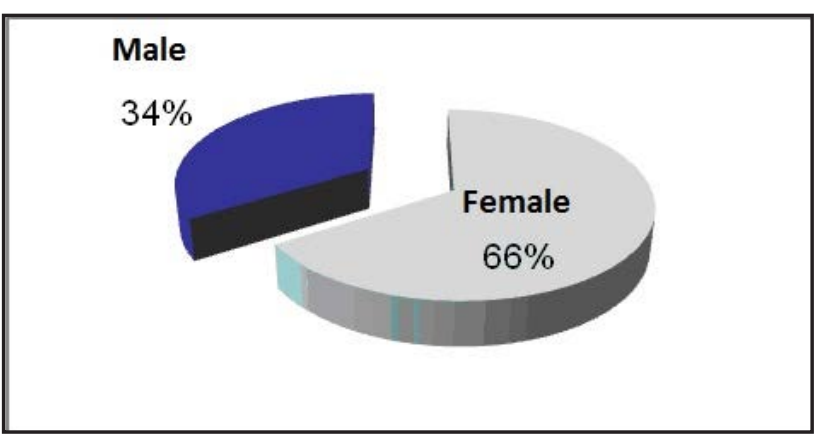

Figure 1 - Gender distribution

The average age of these fractures is over 70 years (Figure 2). The fracture systematization was made using AO classification (Figure 3). It was reported a case of a fracture produced intraoperatory during progression of the nail, 11 bronhopulmonary complications, 5 phlebitis, 7 cases of hematoma after surgery, a case of pseudarthrosis with loosening of implant and 4 dead. There were no septic complication.

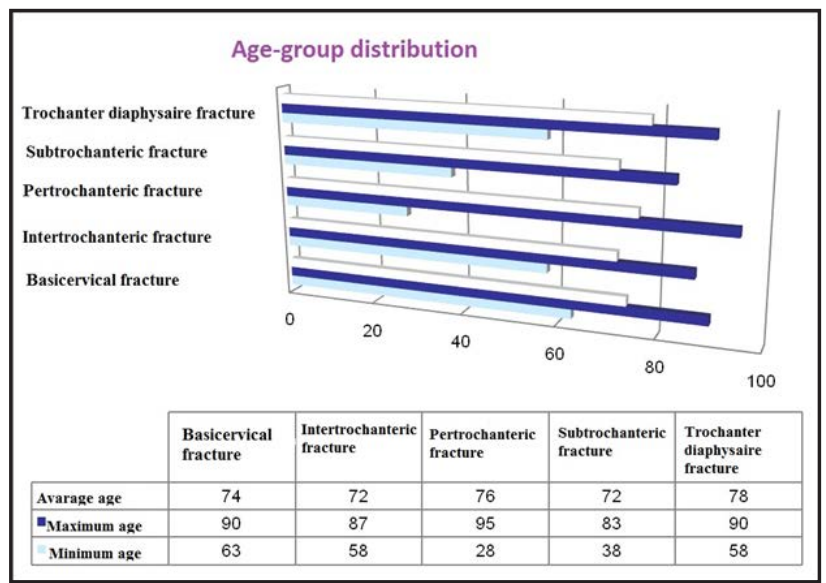

Figure 2 - Age distribution related with the fracture type

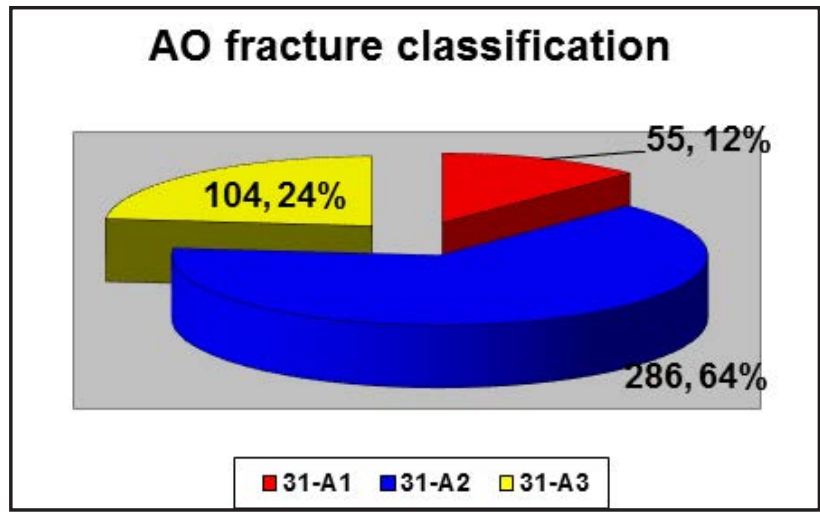

Figure 3 - AO classification of pertrochanteric fractures
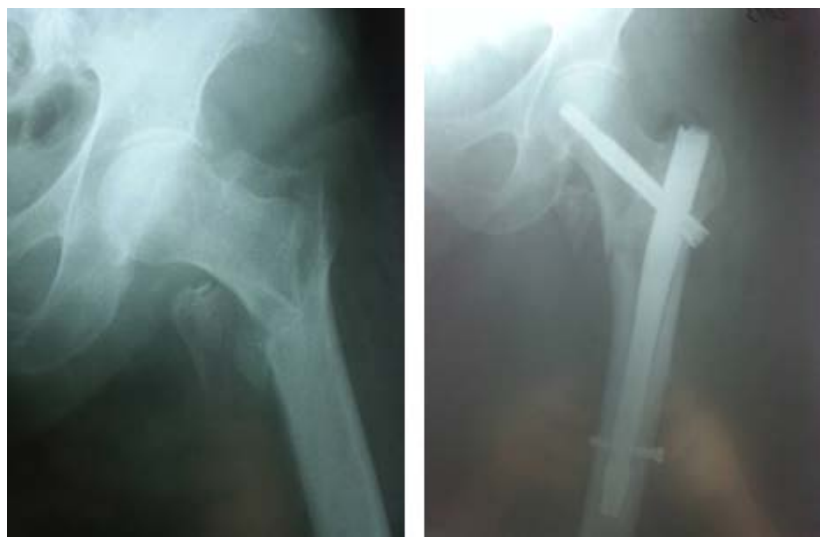

Figure 4 - 31-A2 fracture, 76 years 

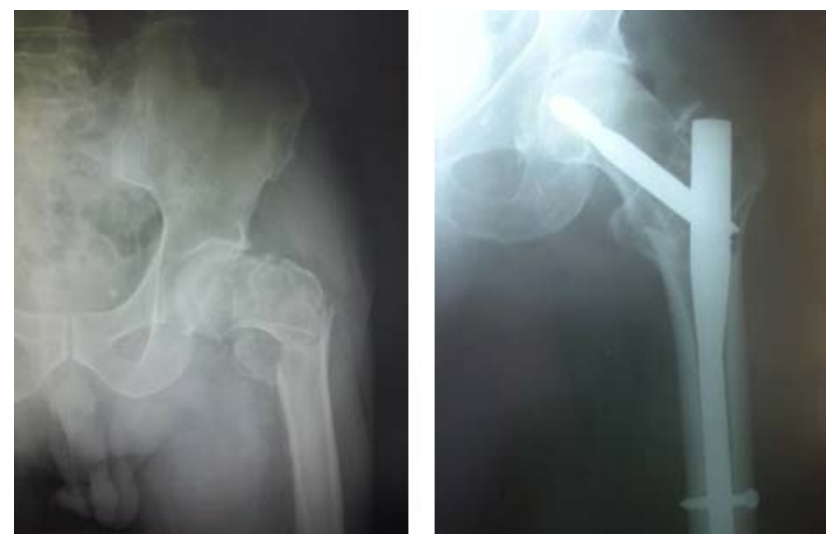

Figure 5 - 31-A2 fracture, 86 years
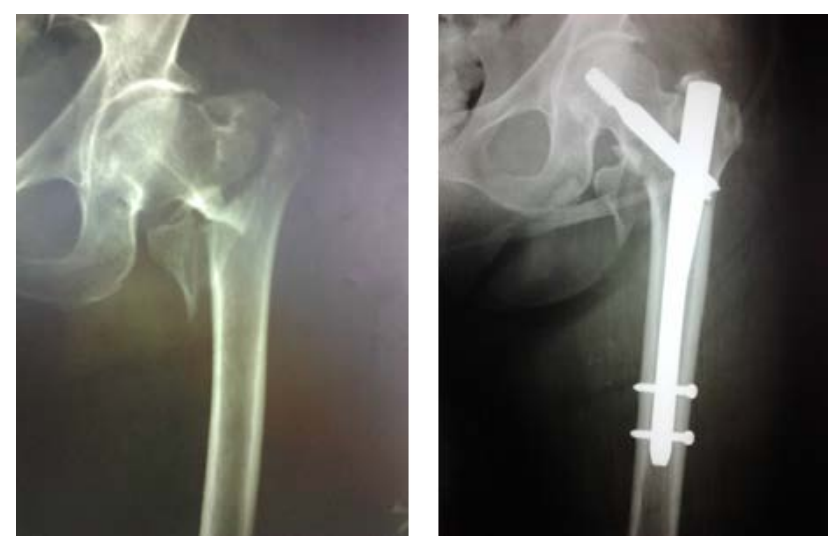

Figure 6 - 31-A3 fracture, 81 years
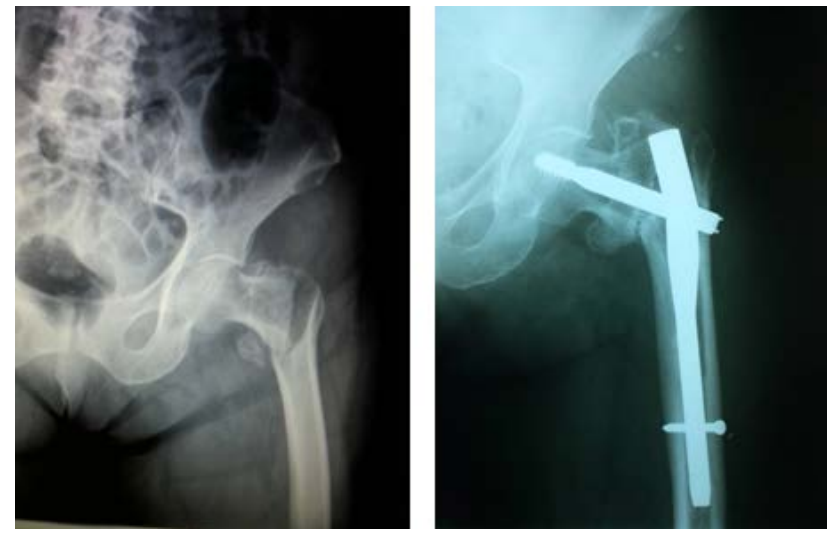

Figure 7 - Pseudarthrosis, loose of implant

\section{Discussions}

The predominant mechanism of hip fracture in the elderly population is a fall from a standing position People with osteoporosis often have other comorbidities that lead to an increased rate of falling. The elderly are also more likely than the general population to experience the type of fall that results in a hip fracture. People with a slower gait have less forward momentum. Thus, when these people fall, they tend to buckle and fall to the side, making a fracture is more likely. The decrease in bone mass in the elderly is caused by a number of factors, including: reduced biosynthetic and replicative potential of osteoblasts, increased osteoclast activity, reduced physical activity (a stimulus for bone remodeling), genetic predisposition, decreased calcium intake, and hormonal influences. The net result is that bone resorption outpaces bone building. Postmenopausal women are especially at risk because of estrogen deficiency. Women can lose as much as $35 \%$ of their cortical bone and $50 \%$ of their trabecular bone in the 30 to 40 years after menopause $[4,5,6,7,8]$.

In present-day surgical practice, it is important to know whether a fracture is stable or unstable: The answer to this question will guide the reduction technique, the type of fixation to be used, and the postoperative management. A good classification must provide information on the fracture's potential of being anatomically reduced, with good apposition of the fragments. Also, it should be possible to tell, in the light of the classification, whether a particular fracture is likely to become secondarily displaced after fixation; this information must be available before the patient is allowed to weight-bear $[9,10]$.

Two factors must be considered in the assessment of stability: loss of medial support, as a result of a separation of the lesser trochanter in association with a fracture of the medial arch; and comminution of the posterior cortex, which is frequently associated with a separation of the greater trochanter. The fracture must be reduced in internal rotation, to close the anterior gap and to replace the posterior cortical fragments $[11,12]$. 
Operative treatment for trochanteric fractures in elderly patient can be a challenging problem. The primary goal of the orthopedic surgeon is to stabilize the fracture so as to permit early mobilization of the patient in hopes of lessening the morbidity and mortality statistics associated with these maladies. Furthermore, in order to prevent the social consequences of loss of ambulatory status, the goal is to return the patient to his prefracture function as quickly as possible, which also addresses the economic burden that hip fractures place on our society.

The Gamma nail osteosynthesis ensure the fracture stability, if we make the anatomic reconstruction of the internal and posterior cortical bone, and the implant was placed correctly. The locomotor recovery must be coordinated in relation with the osteosynthesis quality and the degree of fracture instability.

The defective presurgical evaluation of the fracture stability is the principal cause of the unfavourable results and the failure of osteosynthesis. The anatomic reconstruction of the trochanteric region and the choosing of an implant proper to the fracture can realise the neutralization of the varus and rotation forces, ensuring a correct consolidation.

\section{Conclusions}

The modern treatment of these kinds of fractures must consist of the using of Gamma nail that offers superior advantages comparatively with other types of osteosynthesis. The basic principle of the treatment of these fractures is anatomic reduction and stable fixation.

The Gamma nail combines the features of intramedullary nailing with the compression lag screw and adds the capability of distal locking. Its design and biomechanical principles achieve greater strength and stability for load sharing and physiologic stress. The nailing is accomplished in a closed manner, which should decrease anesthesia time, surgical time, length of exposure, and blood lose.

The stability of the gamma nail permit early mobilization and this nail is golden standard for pertrochanteric fractures.

\section{References}

1. Kaplan, K., Miyamoto, R., Levine, B.R., Egol, K.A. \& Zuckerman, J.D. (2008). Surgical management of hip fractures: an evidence-based review of the literature. II: intertrochanteric fractures. J Am Acad Orthop Surg. 16(11), 665673

2. Bhandari, M., Schemitsch, E., Jönsson, A., Zlowodzki, M. \& Haidukewych, G.J. (2009). Gamma nails revisited: gamma nails versus compression hip screws in the management of intertrochanteric fractures of the hip: a metaanalysis. J Orthop Trauma. 23(6), 460-464

3. Anglen, J.O., Baumgaertner, M.R., Smith, W.R., Tornetta, I.P. \& Ziran, B.H. (2008). Technical tips in fracture care: fractures of the hip. Instr Course Lect. 57, 17-24

4. Della Rocca, G.J., Moylan, K.C. \& Crist, B.D. et al. (2013). Comanagement of geriatric patients with hip fractures: a retrospective, controlled, cohort study. Geriatr Orthop Surg Rehabil. 4(1), 10-15

5. Bojan, A.J., Beimel, C. \& Speitling, A. et al. (2010). 3066 consecutive Gamma Nails. 12 years experience at a single centre. BMC Musculoskelet Disord. 11, 133

6. Cauley, J.A., Lui, L.Y., Genant, H.K., Salamone, L., Browner, W. \& Fink, H.A. et al. (2009). Risk factors for severity and type of the hip fracture. $J$ Bone Miner Res. 24(5), 943-955

7. Gotfried, Y. (2004). The lateral trochanteric wall: a key element in the reconstruction of unstable pertrochanteric hip fractures. Clin Orthop Relat Res. (425), 82-86

8. Mears, S. Fixing hip fractures. Retrieved from http://www.hopkinsmedicine.org/gec/series/ 
fixing_hip_fractures

9. Lichtblau, S. (2008). The unstable intertrochanteric hip fracture. Orthopedics. 31(8), 792-797

10. Carr, J.B. (2007). The anterior and medial reduction of intertrochanteric fractures: a simple method to obtain a stable reduction. $J$ Orthop Trauma. 21(7), 485-489

11. Rockwood, C.A., Green, D.P. \& Bucholz, R.W. (1991). Fractures. Vol 2, 3rd ed. (pp.1538-1560). Philadelphia, Pa: Lippincott Williams \& Wilkins

12. Mabesoone, F. Classification of Trochanteric Fracture patterns. Retrieved from http://www. maitrise-orthopedique.com/corpusmaitri/ orthopaedic/mo65_trochanteric_fracture/index. shtml 